\title{
LOW-FREQUENCY MAGNETIC FIELD ATTENUATION OF ADVANCED COMPOSITE CYLINDERS
}

\author{
Cheng-Nan Chiu, Ming-Shing Lin, and Chun Hsiung Chen \\ Department of Electrical Engineering \\ National Taiwan University \\ Taipei, Taiwan, ROC
}

$\underline{\text { Abstract }}$

The low-frequency magnetic field attenuation of fiberreinforced laminated composite cylinders is analyzed, based on a model which treats the composite cylinder as a multi-layered enclosure and then assumes that each layer is homogeneous and anisotropic. In this study, an analogy between isotropic and anisotropic cylinders is derived. Based on the analogy relationship, numerical results for magnetic field attenuation are presented for $\left[0^{\circ} / 90^{\circ}\right]$ graphite/epoxy cylinders. In particular, the parameters to influence the magnetic field attenuation, such as the geometry and material properties, are investigated in detail.

\section{Introduction}

Advanced composite materials are attractive as substitutes for metals in aircraft applications due to their superior properties in strength-to-weight and modulus-to-weight ratios. Specifically, graphite/epoxy (G/E) and boron/epoxy (B/E) fiber-reinforced composites are increasing used in modern aircraft systems. Because of their anisotropic and marginal conductivity [1], the study of their shielding properties becomes an important issue in electromagnetic compatibility. This study is mainly concerned with the shielding properties of a cylindrical enclosure made of advanced composite material.

Exact analysis of electromagnetic field penetration problem is only possible for the ideal enclosures of simple geometry such as infinite plane, infinite cylinder, and sphere [2]. The procedures are complicated and not easy to extend to the anisotropic and laminated cases. However, the shielding properties of an enclosure are relatively easy to deal with by the circuit approach [3] in low-frequency region.

The purpose of this paper is to present a model for calculating the magnetic field attenuation (MFA) of the advanced composite cylinders. Here, we choose the MFA because the magnetic field penetration is much more serious [4]. The model developed in this study is based on the analogy between the transition equation for laminated isotropic cylinder and that for laminated anisotropic one. Based on this model, numerical results for the $\left[0^{\circ} / 90^{\circ}\right] \mathrm{G} / \mathrm{E}$ cylinder are presented.

\section{Statement of problem}

In this study, the fiber-reinforced laminated composite cylinder is investigated. Each lamina of the cylinder contains a one-dimensional array of fibers which are embedded in an epoxy matrix. Here, the fibers are made from graphites which principally determine the conductivity of the composite, since the epoxy matrix is essentially non-conductive. Shown in Fig. 1 is a typical composite cylinder with orientation $\left[0^{\circ} / 90^{\circ}\right]$ in which the fibers in alternate layers are oriented at right angles to each other.

For the $\left[0^{\circ} / 90^{\circ}\right]$ graphite/epoxy (G/E) cylinder, a pair of adjacent laminas could be regarded as a single layer with thickness $d_{n}$ (Fig. $1(\mathrm{a})$ ) and the layer is then treated as a uniaxially anisotropic and homogeneous medium [5]. In this study, the MFA is defined as the ratio between the surface magnetic field $H\left(\rho_{o}, \omega\right)$ outside the cylinder and that $H\left(\rho_{N}, \omega\right)$ inside the cylinder

$$
M F A=20 \log _{10}\left|\frac{H\left(\rho_{o}, \omega\right)}{H\left(\rho_{N}, \omega\right)}\right|
$$

\section{Analogy between isotropic and anisotropic cylinders}

The composite cylinder to be considered (Fig. 1 (b)) is made up of $\mathrm{N}$ uniaxially anisotropic and homogeneous layers whose thicknesses need not be equal. From the Maxwell's equations, in each layer, we have

$$
\begin{gathered}
\nabla \times \mathbf{E}=-S \overline{\bar{\mu}} \cdot \mathbf{H} \\
\nabla \times \mathbf{H}=S \overline{\bar{\epsilon}} \cdot \mathbf{E}
\end{gathered}
$$

where $\mathrm{S}$ is $j \omega$. The permeability and complex permittivity tensors are

$$
\begin{gathered}
\overline{\bar{\mu}}=\left[\begin{array}{lll}
\mu_{\rho \rho} & \mu_{\rho \phi} & \mu_{\rho z} \\
\mu_{\phi \rho} & \mu_{\phi \phi} & \mu_{\phi z} \\
\mu_{z \rho} & \mu_{z \phi} & \mu_{z z}
\end{array}\right] \\
\overline{\bar{\epsilon}}=\left[\begin{array}{lll}
\epsilon_{\rho \rho}+\frac{\sigma_{\rho p}}{S} & \epsilon_{\rho \phi}+\frac{\sigma_{\rho \phi}}{S} & \epsilon_{\rho z}+\frac{\sigma_{\rho z}}{S} \\
\epsilon_{\phi \rho}+\frac{\sigma_{\phi p}}{S} & \epsilon_{\phi \phi}+\frac{\sigma_{\phi \phi}}{S} & \epsilon_{\phi z}+\frac{\sigma_{\phi z}}{S} \\
\epsilon_{z \rho}+\frac{\sigma_{z \rho}}{S} & \epsilon_{z \phi}+\frac{\sigma_{z \phi}}{S} & \epsilon_{z z}+\frac{\sigma_{z z}}{S}
\end{array}\right]
\end{gathered}
$$

where $\mu$ is the permeability, $\epsilon$ is the permittivity, and $\sigma$ is the conductivity.

By considering the $e^{-j n \phi}$ and $e^{-j k z}$ dependences along $\phi$ and $z$ directions and assuming that $\overline{\bar{\epsilon}}$ and $\overline{\bar{\mu}}$ are independent of $\phi$ and $z$, Eqs. (2) and (3) can be simplifed to

$$
\frac{d \mathbf{U}}{d \rho}=\mathbf{G}(\rho) \cdot \mathbf{U}
$$

where $\mathbf{U}$ is $\left[\begin{array}{llll}E_{z} & E_{\phi} & H_{z} & H_{\phi}\end{array}\right]^{t}$ and $\mathbf{G}(\rho)$ is a complicated $4 \times 4$ matrix. 
Eq.(6) can further be simplified by using the following assumptions: 1) the external field is assumed to be uniform; 2) the frequency is in quasi-static range; 3 ) the thickness of each layer is electrically very thin and also much smaller than the radius; 4) the electromagnetic properties of the G/E composite could be regarded as a uniaxially anisotropic material. By these assumptions and using the finite-difference algorithm [6], the relationship between internal and external magnetic fields of each layer could be expressed as

$$
\mathbf{U}\left(\rho_{n}\right)=\left[\mathbf{I}-d_{n} \mathbf{G}\left(\rho_{n-1}\right)\right] \cdot \mathbf{U}\left(\rho_{n-1}\right)
$$

where the subscripts " $n$ " and " $n-1$ " are associated with the radii inside and outside the nth layer, respectively, and $d_{n}$ is the thickness of the nth layer. Here $I$ is the identity matrix and the simplified $\mathbf{G}\left(\rho_{n-1}\right)$ is

$$
\mathbf{G}\left(\rho_{n-1}\right)=\left[\begin{array}{cccc}
0 & 0 & 0 & S \mu_{n-1} \\
0 & \frac{1}{\rho_{n-1}} & -S \mu_{n-1} & 0 \\
0 & -\sigma_{n-1}^{t} & 0 & 0 \\
\sigma_{n-1}^{t} & 0 & 0 & \frac{-1}{\rho_{n-1}}
\end{array}\right]
$$

where the superscript " $t$ " means the transverse directions ( $\phi$ and $z$ ). From (7), the relationship between internal and external magnetic fields of nth layer may be expressed by the transition equation

$$
H_{z}\left(\rho_{n-1}\right)-H_{z}\left(\rho_{n}\right)=\sigma_{n-1}^{t} d_{n} E_{\phi}\left(\rho_{n-1}\right) .
$$

On the other hand, we consider an isotropic cylinder of the same size with anisotropic layers be replaced by the isotropic ones. By using circuit approach [3] for such a cylinder, we can obtain the relationship between internal and external magnetic fields as

$$
H_{z}\left(\rho_{n-1}\right)-H_{z}\left(\rho_{n}\right)=\sigma d_{n} E_{\phi}\left(\rho_{n-1}\right) .
$$

By a comparison of (10) with (9), we can establish the analogy between isotropic and uniaxially anistotropic cylinders as

$$
\begin{array}{cc}
\text { isotropic } & \text { uniaxially anisotropic } \\
\sigma & \sigma_{n-1}^{t} \\
\mu & \mu_{n-1} .
\end{array}
$$

Thus, once the ratio between the fields outside and inside an isotropic cylinder has been derived, the ratio for a uniaxially anisotropic layer of the same size could be computed by the analogy.

For a multi-layerd cylinder with isotropic and homogeneous metal in each layer, we may propose a circuit model to calculate its MFA. The magnetic field ratio may be expressed as

$$
\frac{H\left(\rho_{o}\right)}{H\left(\rho_{N}\right)}= \begin{cases}1+\sum_{n=1}^{N} \frac{S L_{n}}{A_{n}}, & \delta_{n} \gg d_{n} \\ \left\{1+\sum_{n=1}^{N} \frac{S L_{n}}{R_{n}} \cdot \frac{\delta_{n}}{(1+j) d_{n}}\right\} \frac{\exp \left[(1+j) \sum_{n=1}^{N} d_{n} / \delta_{n}\right]}{2 N}, & \delta_{n} \ll d_{n}\end{cases}
$$

where $N$ is the total number of layers. Here, $\delta_{n}$ and $d_{n}$ are the skin depth and thickness of the nth layer, respectively, and

$$
\frac{L_{n}}{R_{n}}=\frac{\sigma_{n} d_{n} \mu_{o} \rho_{N}}{2}+\sum_{i=n+1}^{N} \frac{\sigma_{n} \mu_{i} d_{n} d_{i} \rho_{i-1}}{\rho_{n}}
$$

Based on the analogy given in (11), we can predict the MFA of multi-layered uniaxially anisotopic cylinders by using
Eqs. (11) and (12)

\section{Numerical results}

For the $\left[0^{\circ} / 90^{\circ}\right] \mathrm{G} / \mathrm{E}$ cylinder shown in Fig. 1, we choose $d_{1}=d_{2}=\ldots=d_{N} \equiv d$. The transverse conductivity $\sigma^{t}$ of the $\mathrm{G} / \mathrm{E}$ composite is $10^{4} \mho / \mathrm{m}$, and the permittivity $\epsilon^{t}$ can be neglected in the quasi-static range.

A comparison of our results with those from Birken's paper [7] for 4-layer G/E cylinder is shown in Fig. 2. Good agreement between both results confirms the accuracy of the proposed model. Also included in Fig. 2 are the results of infinite plane structure for comparison. It shows that the infinite plane approximation of cylinder could be extremely overestimated in low-frequency region.

Presented in Fig. 3 is the relationship between the MFA and the ratio of thickness $d$ to skin depth $\delta$ with $\mathrm{N}$ (number of layers) as parameters. Obviously, one may improve the MFA by increasing the number $\mathrm{N}$ as the ratio $\rho_{N} / d$ is fixed. Fig. 4 shows the same relationship but with the ratio $\rho_{N} / d$ as parameters. It is found that the larger the ratio $\rho_{N} / d$, the better the MFA of the cylinder.

Shown in Fig. 5 is a comparison of MFA among aluminum, titanium, and G/E cylinders with the same total thickness. Apparently, the G/E cylinder has the poor shielding performance than that of metal cylinders.

\section{Conclusion}

In this study, the analogy between multi-layered isotropic cylinder and uniaxially anisotropic cylinder has been derived. Based on this analogy relationship, the MFA of a uniaxially anisotropic cylinder can be computed. Finally, the numerical results of such a cylinder have been discussed.

The MFA is a representation of shielding performance against magnetic field. Particularly, it depends on many parameters such as the layer thickness to skin depth ratio, the radius to layer thickness ratio, and the number of layers. Especially, one may improve the MFA by increasing one of those parameters with the others fixed.

All the results of this investigation are derived under the low-frequency assumptions, thus internal resonance of the cylinder is not significantly excited. In high-frequency range, internal resonance is important and worthy of further study.

\section{References}

[1 ] L. Allen et al., "An investigation of the electromagnetic properties of advanced composite materials," IEEE International Symp. on Electromagn. Compat., pp. 174-179, Washington, 1976.

[2 ] K. S. H. Lee and G. Bedrosian, "Diffusive electromagnetic penetration into metallic enclosures," IEEE Trans. 
Antennas and Propagat. vol. AP-27, pp. 194-198, Mar. 1979.

[ 3 ] J. E. Bridges, "An update on the circuit approach to calculate shielding effectiveness," IEEE Trans. Electromagn. Compat., vol. 30, no. 3, pp. 211-221, Aug. 1988.

[ 4 ] L. O. Hoeft and J. S. Hofstra, "Experimental and theoretical analysis of the magnetic field attenuation of enclosures," IEEE Trans. Electromagn. Compat., vol. 30, no. 3, pp. 326-340, Aug. 1988.

[ 5 ] K. F. Casey, "Advanced composite material and electromagnetic shielding," IEEE International Symp. on Electromagn. Compat., pp. 228-232, Atlanta, GA, June 20-22, 1978.

[ 6 ] W. C. Chew, Waves and Fields in Inhomogeneous Media, New York: Van Nostrand Reinhold, 1990. ch. 2, pp. 131133.

[ 7 ] J. A. Birken et al., "Advanced composite aircraft electromagnetic design and synthesis," IEEE International Symp. on Electromagn. Compat., pp. 562-569, 1981.

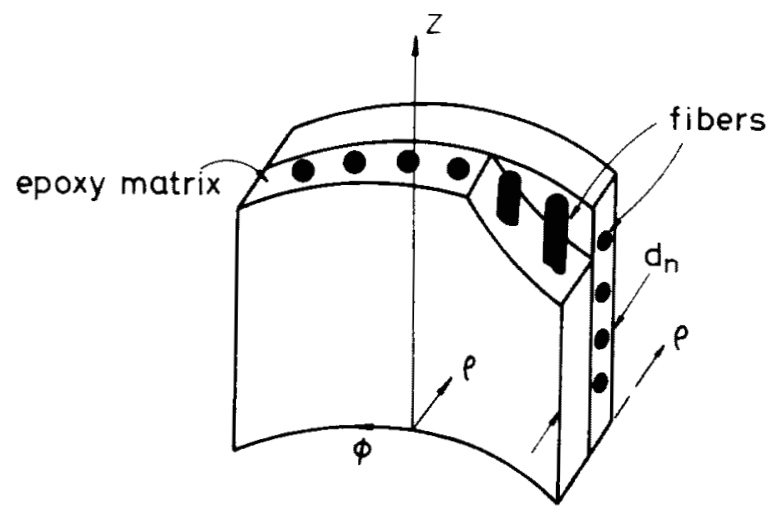

(a)

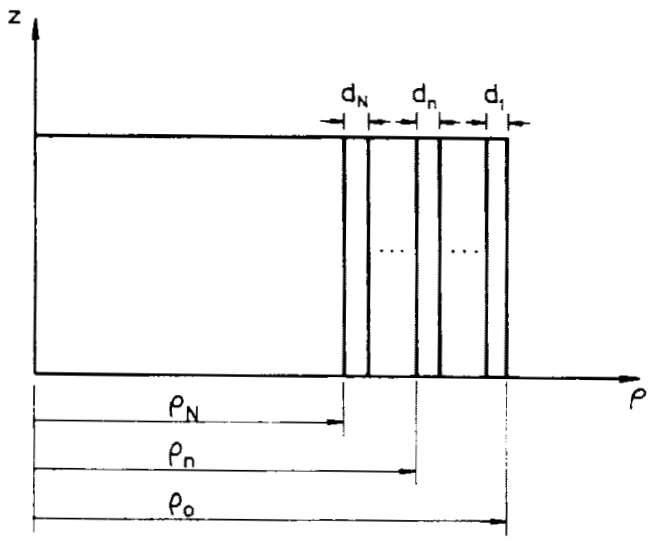

(b)

Fig. 1 Geometry of $\left[0^{\circ} / 90^{\circ}\right]$ graphite/epoxy cylinder. (a) A pair of adjacent laminas. (b) Cross-sectional view of the cylinder.

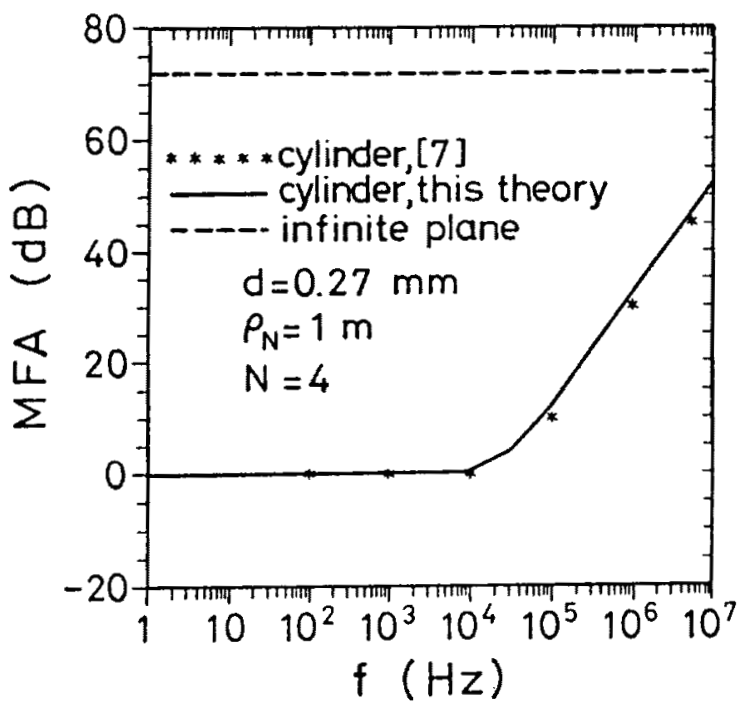

Fig. 2 A comparison of our results with those of [7] and those from infinite-plane structure.

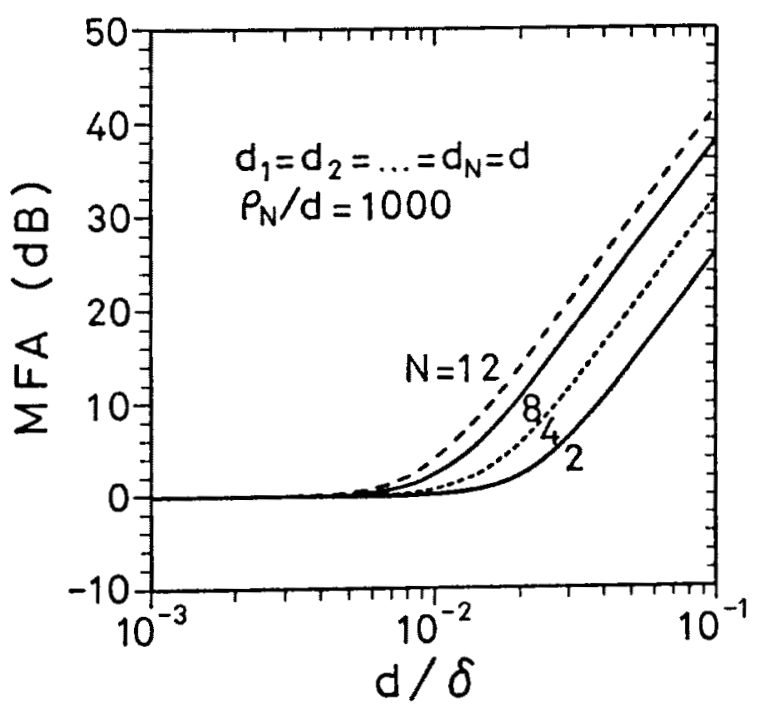

Fig. 3 MFA of $\left[0^{\circ} / 90^{\circ}\right] \mathrm{G} / \mathrm{E}$ cylinders with $\mathrm{N}$ as parameters. 


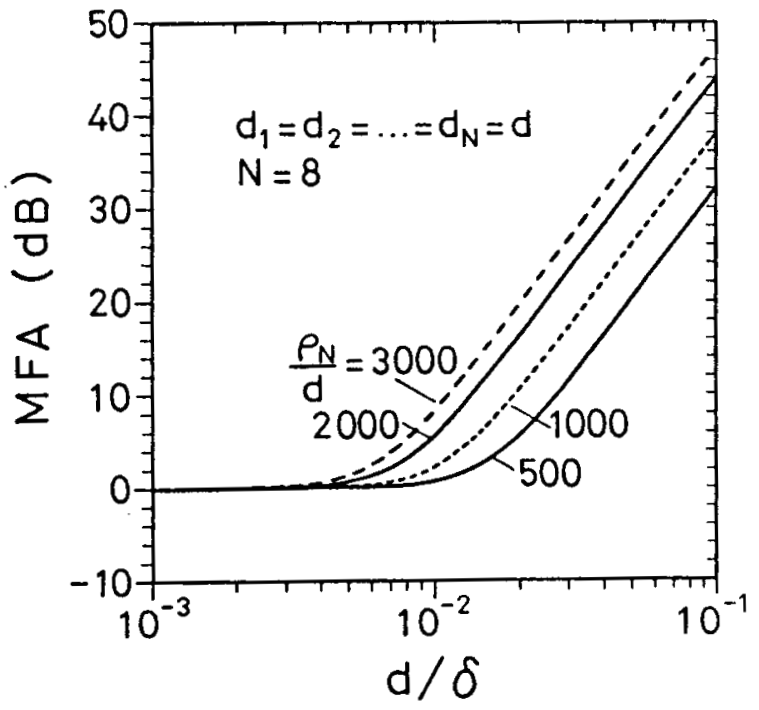

Fig. 4 MF'A of $\left[0^{\circ} / 90^{\circ}\right] \mathrm{G} / \mathrm{E}$ cylinders with $\rho_{N} / d$ as parameters.

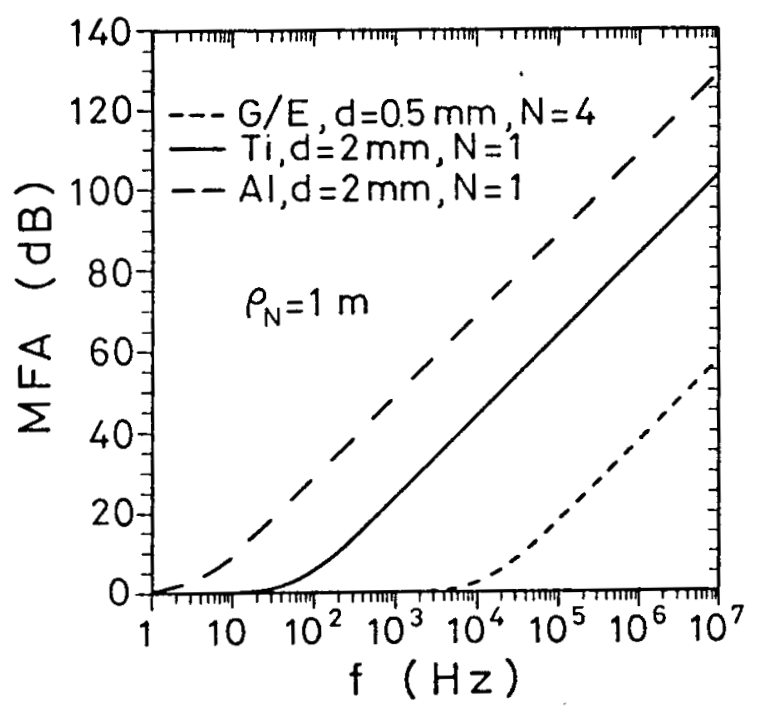

Fig. 5 Comparison of shielding performance among different materials. 